\title{
Experimental and Numerical Investigation of a Compressor Cascade at Highly Negative Incidence
}

\author{
P K Zachos*, N Grech, B Charnley, V Pachidis, R Singh \\ Cranfield University, School of Engineering, Department of Power and Propulsion, Gas Turbine Performance \\ Engineering Group, Cranfield, Bedfordshire, MK43 OAL, UK \\ ${ }^{*}$ Address all correspondence to this author. E-mail:p.zachos@cranfield.ac.uk
}

\begin{abstract}
The performance prediction of axial flow compressors and turbines still relies on the stationary testing of blade cascades. Most of the blade testing studies are done for operating conditions close to the design point or in off-design areas not too far from it. However, blade - and consequently engine - performance remain unexplored at relatively far off-design conditions, such as windmilling or sub-idle. Such regimes are dominated by blade operation under extremely low mass flows and rotational speeds that imply highly negative values of incidence angle, thus totally separated flows on the pressure side of the blades. Those flow patterns are difficult to be measured and even more difficult to be numerically predicted as the current modelling capability of separated internal flows is of limited reliability. In this paper, the performance of a 3-dimensional linear compressor cascade at highly negative incidence angle is initially experimentally investigated. The main objective of the study is to derive the total pressure loss and outlet flow angle through the blades and use the data for the validation-calibration of a numerical solver enhancing its capability to predict highly separated flows. The development of the CFD model and the simulation strategy followed are also presented. The numerical results are compared against the derived test data demonstrating a good agreement. In addition, most trends of the properties of interest have been captured sufficiently, therefore the physical phenomena are considered to be well captured, allowing the numerical tool to be used for further studies on similar test cases.
\end{abstract}

Keywords: Compressor cascade, negative incidence, separated flow, experimental, numerical

\section{INTRODUCTION}

The experimental verification of any design intent, as far as turbomachinery blading is concerned, is considered to be a very significant step before the integration of a blade design into a gas turbine engine. Traditionally, cascades of blades in linear or annular configuration were employed for the experimental understanding of the complex flow phenomena and the derivation of their performance under various operating conditions (Todd, 1947). A huge range of instrumentation approaches as well as of wind tunnel configurations can be recorded throughout the literature, implying the depth of the experimental studies performed by different research groups (Carter, 1950 and Sieverding, $1985)$ in this direction. The result of all the extensive studies carried out, especially before the computational revolution, is a large amount of experimental data for different blade families under various conditions, which are currently utilised as a validation platform for more sophisticated predictive methods of numerical or analytical nature (Lieblein, 1965). From a slightly different perspective, analytical expressions derived from the experimental data, mainly correlating the pressure loss through the blade channels with the operating conditions, are extensively built into numerical tools, enhancing their reliability-predictability (Lieblein, 1959 and Lieblein, 1956). However, as the major concern of a turbomachinery blade designer is to verify whether a newly designed component performs as it is supposed to do, the majority of the experimental studies carried out, is focused on the design point performance of the blades, while a narrow off-design range of operation is often a topic of research as well (Howell, 1945, Dixon, 1998, Korakianitis, 1993). The latter is reflected in the actual range of the flow incidence angles under which a cascade of blades is experimentally tested which is never wider than $+10-23$ degrees in respect to the design point operation that is the operation at zero incidence (Howell, 1942).

In cases that far off-design areas of blade operation are being investigated, additional experimental studies have to be carried out. These usually aim at understanding initially the dominant flow phenomena occurring and furthermore to describe quantitatively the flow properties such as the pressure change or the deflection angles downstream of the blades. As frequently mentioned within the literature (Kurzke, 1996, Riegler et al., 2000, Alexiou, 2007), multiple benefits can arise from such kinds of studies. Understanding the flow through blades at extremely high incidence enhances the component performance prediction methods but also the whole engine performance simulation for far off-design power settings such as the sub-idle relight transient maneuvers (windmill altitude and quick relights) or groundstarts. Additionally, the pressure loss modeling can be significantly enhanced with important benefits on the numerical tools where pressure loss models are employed (Novak, 1967, Pachidis et al., 2006). Finally, validationcalibration of CFD codes for predicting the extremely challenging areas of separated flows can be also carried out (Pachidis et al., 2006). 
The increased computational power available nowadays throughout the research community has increased the application of high-fidelity numerical approaches on every kind of flow prediction, starting from pure aerodynamic phenomena occurring at inlets or compressors and extending them in combustion or turbine cooling research, employing multidisciplinary tools of chemical or heat transfer nature (Chaluvadi et al., 2003, Ramakrishna et al., 2009, Ummiti et al., 2009). In case of far off-design turbomachinery performance studies such as the above-described ones, the availability of experimental datasets is the only aspect that prevents the numerical tools from being further validated and at a second stage further improved. More specifically, the lack of experimental data regarding totally separated internal flows through compressor passages is quite limited, whichwhen combined with the complexity of the flow patternsprevents numerical studies from being further employed since they remain partially validated. Obtaining confidence on the numerical solvers, can significantly enhance the predictive capability regarding compressor performance during the starting or relight processes of a gas turbine engine, whereas important contribution can be also made to through-flow simulation tools by extending the applicability of the pressure loss models utilised by them. In overall, reliable numerical predictions of off-design component performance give a valuable insight on the understanding of the flow patterns, while the overall engine modeling capability is also significantly improved minimising at the same time the need for the effectively expensive experimental testing.

This paper describes an experimental study of the flowfield generated by a non-rotating compressor cascade operating at a highly negative incidence angle, which reflects the lower boundary of engine's operation (referred to as 'locked rotor operation' from now on). The results are used as a validation platform for a numerical model, which is hereafer presented as well. The experimental as well the numerical procedures used are described in great detail. The results from both approaches are compared against each other in an effort initially to validate and at a later stage to calibrate the numerical tool enhancing its capability on predicting totally separated flows through compressor cascades operating under severely negative incidence angles.

\section{CASCADE GEOMETRY}

The compressor cascade under examination consists of nine (9) blades in a linear configuration as shown in Fig. 1. The 3-dimensional blade geometry is described in Fig. 2. A similarity between the incidence angle (Fig. 2 bottom right) and inlet metal angle (Fig. 2 top left) distributions can be easily observed and is due to the fact that the velocity triangle at the inlet of a locked rotor consists only of the axial velocity, thus the incidence angle becomes identical with the inlet metal angle definition. The actual formation of the velocity triangle under those conditions is presented in Fig. 3.

It has been decided the blades to be tested in a linear configuration, instead of an annular one, for two main reasons; (i) the actual blade performance is not the primary objective of this investigation since the experimental work has been set up only for calibration-validation purposes of a correspondent numerical model. The latter is utilized to investigate the annular configuration at a later stage, thus (ii) a relatively easier to be designed and manufactured experimental facility was preferred to avoid the complexity of designing a 3-dimensional wind tunnel to accommodate the blades.

Consequently, the cascade results presented hereafter do not necessarily reflect the actual performance of the blades under the specified conditions and they should be only considered as a calibration-validation platform for numerical models simulating similar phenomena.

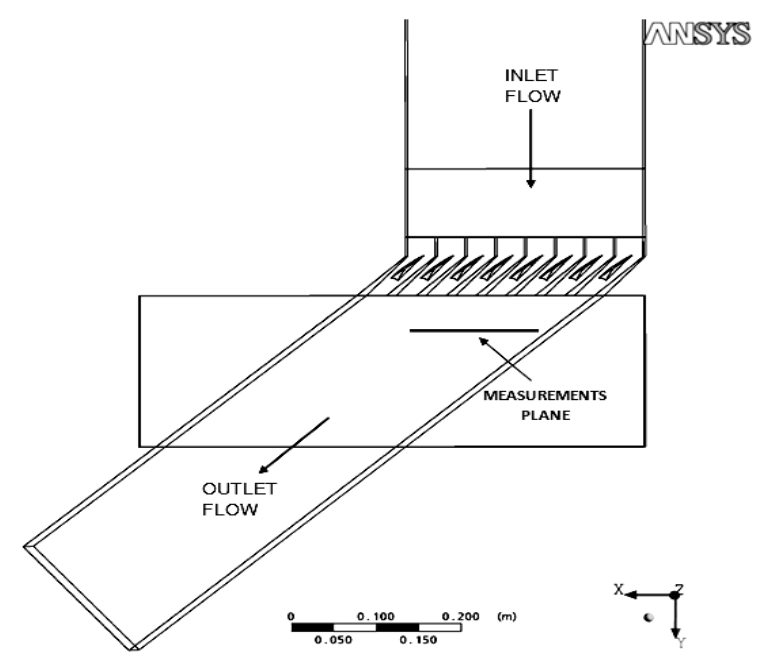

Figure 1 Top view of the compressor cascade domain specifying flow direction and measurement position.

\section{EXPERIMENTAL APPROACH}

The experiments were carried out at Cranfield University's low speed cascade wind tunnel designed for testing blades at high incidence. A schematic layout of the facility is presented in Fig 4.

As seen in the picture, the wind tunnel is a suck down type. Previous wind tunnel designs of different types on which the current configuration is based are given in Gostelow (1984) and Pollard et al. (1967). The intake is followed by the boundary layer suction layers just upstream of the blades, ensuring that no boundary layer effects will affect the upstream flow profiles. The boundary layer mass flow is returned at the fan face. The upstream flow uniformity is ensured thanks to a flow straightener installed just after the convergent part of the inlet. 

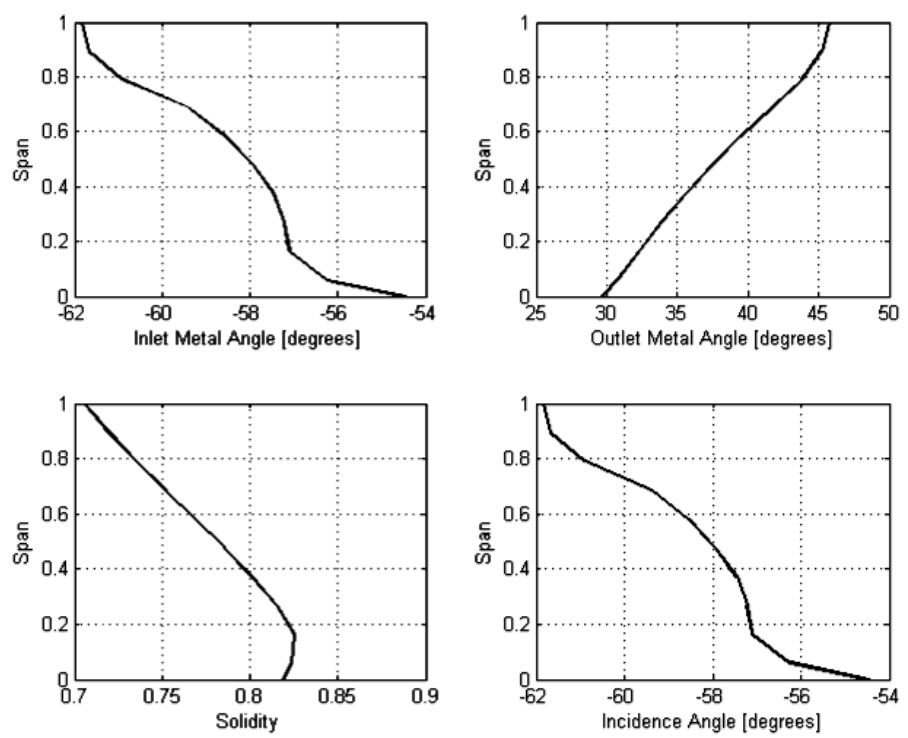

Figure 2 Spanwise distributions of the main blade geometrical features.

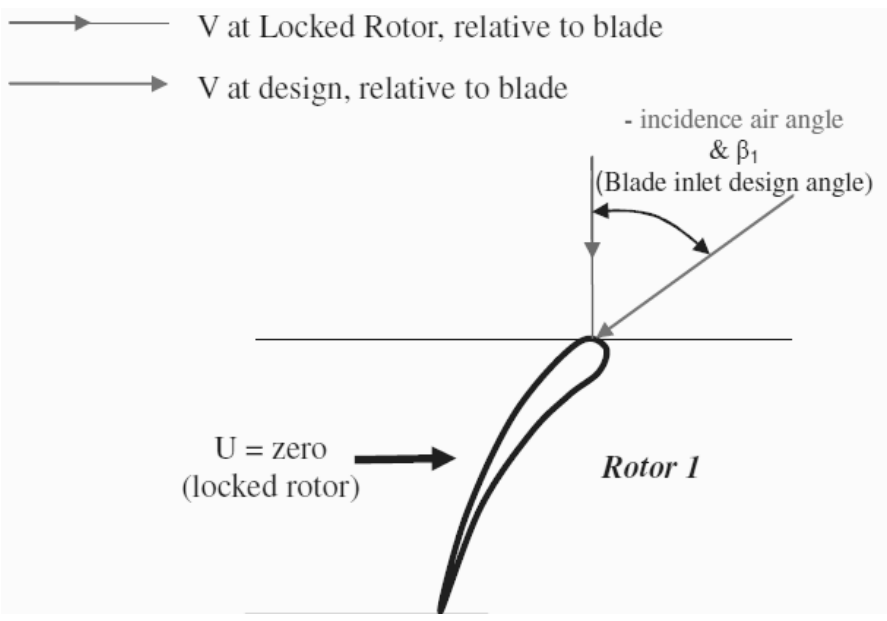

Figure 1 Velocity triangle formulation at locked rotor conditions.

The 9 blade test section comes before a plenum chamber designed to accommodate the outlet flow, aligned with the blade outlet angle at mid span. Preliminary wind tunnel design studies have been carried out using CFD, regarding the definition of the plenum chamber's size and its orientation in order for the wakes to be appropriately accommodated. Flow visualisation tests performed at a later stage proved the suitability of the proposed design. The main valve for mass flow control is positioned before the additional secondary inlet to ensure that the fan operates far enough from its surge point. Separate valves control both the boundary layer suction flows as well as the secondary fan inlet flow. An image of the experimental facility including the instrumentation mounting as well as the data acquisition system is presented in Fig. 5.

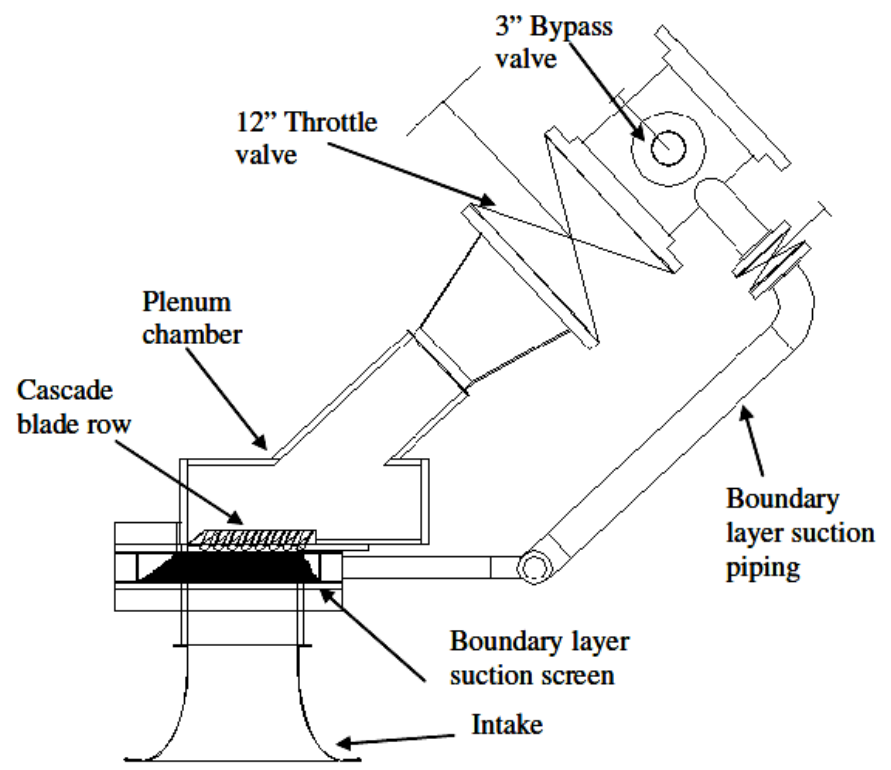

Figure 2 Top view of the experimental facility design.

As far as the instrumentation is concerned, a wide variety of methods to choose from is available. As Johansen et al. (2003) mention, steady state flow measurements are typically conducted with pneumatic probes due to their relatively lower response compared to non-intrusive methods (PIV or LDA) typically employed for capturing unsteady flow patterns at high frequencies (Foerster et al., 2000, Schodl, 1975, Wernet et al., 2001). For this study, since the unsteady flow effects were not of primary importance, two pneumatic cobra probes have been employed; a 3-hole probe for a first assessment while a 5-hole probe was used for the higher fidelity final flow field capturing. Both probes were of $2 \mathrm{~mm}$ diameter, in-house developed, manufactured and calibrated. The measurement position was decided to be located at 2 chords downstream of the blades due to the large separation eddies expected within the wakes (see Fig. 2).

A fully automated data acquisition interface (hardware and software) was developed for conducting the measurements. A schematic diagram of this system is presented in Fig. 6. The data acquisition system was based on an analogue-to-digital card, with a maximum sampling rate of $20 \mathrm{kHz}$ and a 32-bit resolution. The communication between the A/D card and the PC was achieved employing the DAQ driver software. The data acquisition hardware consists of three transducers, one for each hole of the pneumatic probe. The signal from each transducer is fed into an amplifier module with 'low pass' filters to reject noise frequencies. The three amplified signals are then transmitted to the $A / D$ card in separate channels and finally the measured data is monitored and recorded to files via an interface developed in LabView ${ }^{\circledR}$. 


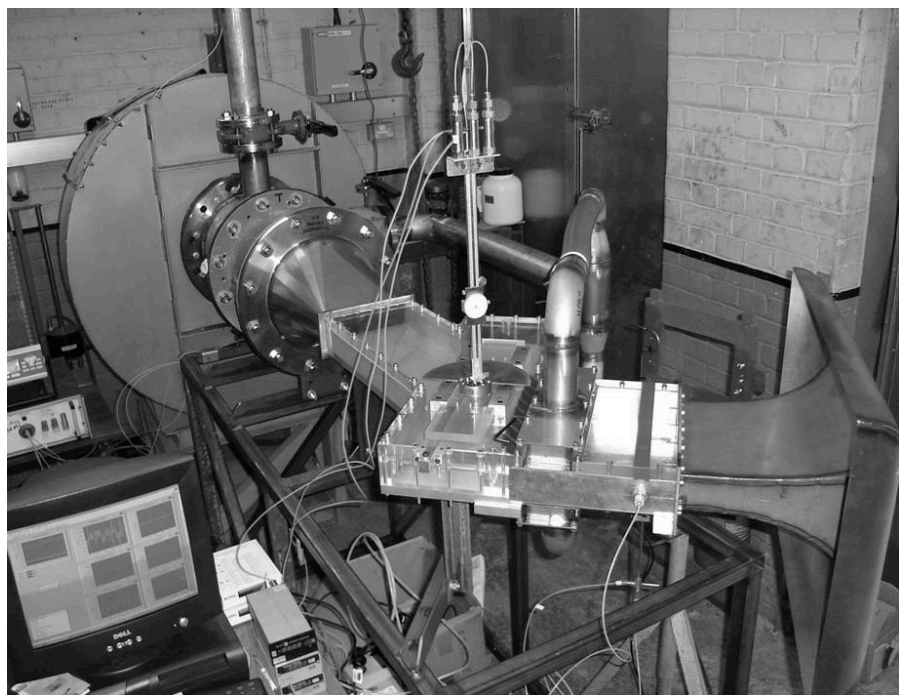

Figure 3 The experimental facility.

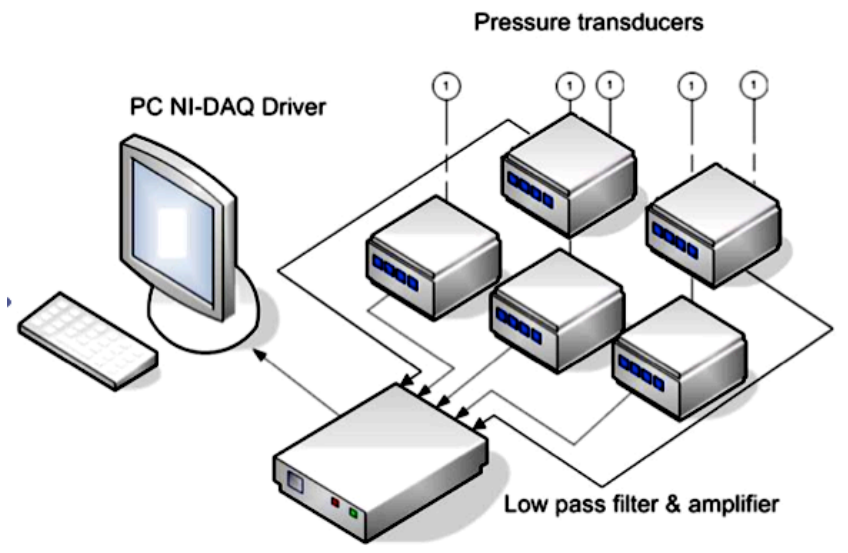

NI AVD Card

Figure 4 Layout of the data acquisition system.

A LabView ${ }^{\circledR}$ function allows the interpretation of the measured information into the flow properties at each point utilising the characteristic maps of the pneumatic probe. Therefore, the output information consists of one file for each measurement position downstream of the blades, where the total and static pressure as well as the 3D velocity vector are recorded as a function of time, also dependent upon the acquisition frequency specified as input to the $A / D$ card.

The measurements have been conducted at three different inlet Mach numbers to identify whether there are any Reynolds number effects. The measurement grid consisted of 598 points per channel arranged on a rectangular grid with $23 \times 26$ points in the pitchwise and spanwise direction respectively. All the tests were run in a steady state approach averaging the measurements in time.
The acquisition frequency was set at $100 \mathrm{~Hz}$ for a time period of 10 seconds.

At all rig power settings the following tests were carried out before conducting the actual measurements in order for the good functionality of the facility to be ensured:

- Inlet duct calibration for deriving the actual mass flow and total pressure loss caused by the upstream section.

- Evaluation of the upstream flow profiles.

- Boundary layer suction valve calibration and performance.

- Flow visualisation in the section downstream of the blades to assess the impact of the plenum chamber on the flowfield.

- Tests without blades to assess potential plenumfan interactions.

It has been established that the flow periodicity downstream of the blades was within an acceptable range, whereas the boundary layer suction valves had to remain at a fully opened position in order to avoid boundary layer growth influence on the upstream flow profiles. Additionally, the flow visualisation tests showed that the flow expands very well in the plenum chamber without any side or back wall interactions.

The uncertainty within the experimental measurements has been investigated according to the methodology proposed by Abernethy et al. (1985) and adapted by the American Society of Mechanical Engineers as a standard uncertainty analysis. The detailed analysis for the 3-hole probe measurements (Zachos et al., 2009) showed that the total and static pressure as well as the velocity yaw angle values have been measured with an error of $0.0041 \mathrm{kPa}$, $0.026 \mathrm{kPa}$ and $0.0079 \mathrm{deg}$ respectively. This analysis implies that the most significant uncertainty band is observed at the static pressure derivation whereas the total pressure and the yaw angle lie within extremely narrow uncertainty bands. This can be explained based on the fact that the velocity fluctuations caused by the unsteadiness of the flow are also reflected onto the static pressure flow field while the total pressure remains in much narrower range since it remains unaffected by time dependent phenomena. In addition, the uncertainty analysis conducted for the 5-hole probe acquired data showed that the pressure indications lie within a similar to the 3-hole probe data error band while the pitch angle error is as much as $0.0063 \mathrm{deg}$.

\section{NUMERICAL APPROACH}

The geometrical model used for the numerical calculations is presented in Fig. 7. It consists of an inlet duct, the cascade section, the plenum chamber and an outlet duct. The dimensions of the model reflect the geometry of the experimental facility. 
All the numerical simulations of this study have been carried out using ANSYS CFX® solver, widely applied for turbomachinery flow predictions. A high resolution (second order) level of accuracy has been chosen for the solution of the RANS equations.

The computational grid has been separately generated for each one of the above-mentioned domains. All the regions, apart from the boundary layer sectors, have been meshed using hexahedral unstructured approach. The $y+$ in every wall has been kept below 20 in such a way that the wall boundary layer could be modelled without employing wall functions. A structured mesh treatment has been employed for the boundary layer sections which was found to be of fundamental importance for predicting the near the wall flow patterns as well as the flow separation. Since the enhanced wall treatment was chosen to be utilized in this study the $y+$ had to be kept below 2 in the blade boundary layer regions. The O-mesh configuration employed for the near the wall region in the blade block is illustrated in Fig. 8.

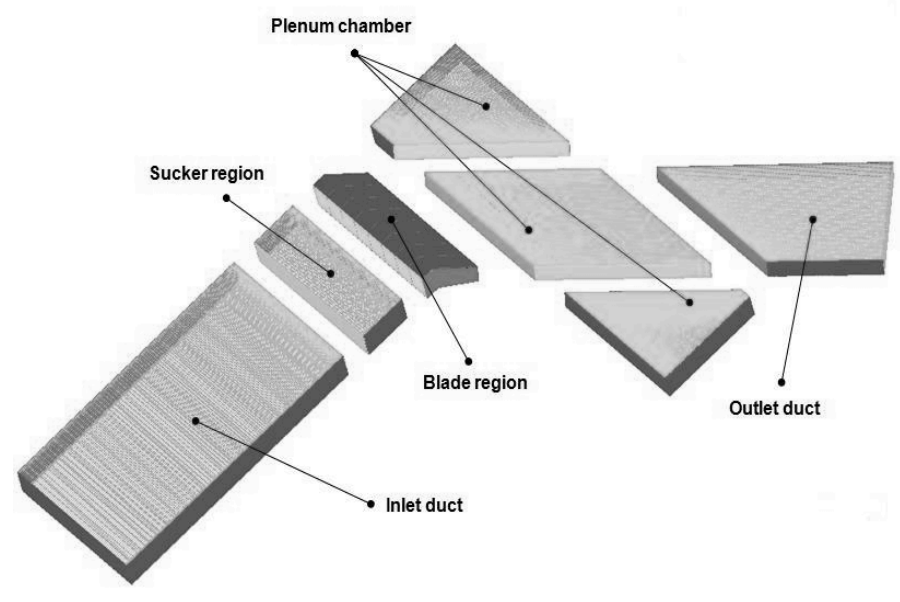

Figure 5 Blocks of the numerical model.

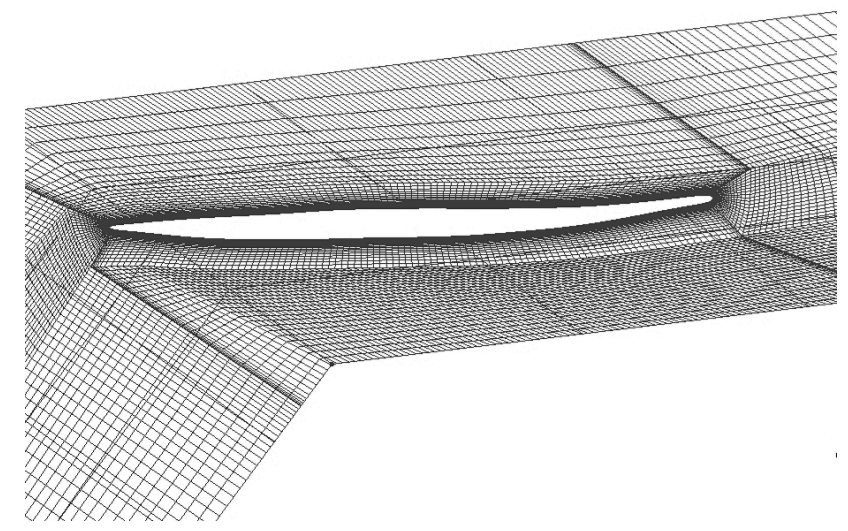

Figure 6 O-mesh for the blade boundary layer regions.

Since one of the most crucial challenges of this numerical study is the robustness of the model and its converging capability, the boundary condition set specified is of great importance. A total pressure at the inlet and a velocity (or mass flow) at the outlet have been applied. Experimental values of the total and static pressure in the region between the blades and the boundary layer suction block are available from the rig calibration process carried out (Zachos et al., 2009). Additionally, as the air's density and the upstream area are known, the mass flow can be easily calculated. In general, the total pressure at the inlet is kept constant in all simulations, while the mass flow rate at the outlet is calculated as mentioned above and varies as a function of the inlet Mach number.

The turbulence closure set is also considered to be of major importance in this case due to the extremely challenging nature of the flow patterns developed around the high incidence blades. The turbulence parametric research is focused on the non-algebraic two-equation eddy-viscosity models which are independent on the difficult to define algebraic length-scales. The most widely used model is the $k-\varepsilon$ model. However, the drawbacks that this model demonstrates (Ekaterinaris et al., 1994) caused poor convergence of the solver. The $k-\omega$ model of Wilcox among all the models developed trying to solve the $k-\varepsilon$ drawbacks, is the one that presents the biggest improvements both in accuracy and robustness (Wilcox, 1988). However it still presents a big drawback, which lies in its strong sensitivity to the free-stream values that are specified outside the shear stress layer therefore employment of more sophisticated turbulence models is required. In that direction the applicability of BSL (Baseline) $k-\omega$ and SST (Shear Stress Transport) $k-\omega$ was investigated. Both of them are based on the simple $k-\omega$ model while some extra care is taken for the boundary layer treatment to increase the performance and the converging capability of the solver. A further description of the model structure is given in Wilcox (1988) whereas studies on the most suitable turbulence closures can be also found in Menter (1993) and Menter (1994).

The SST $k-\omega$ model was found to perform well in the current test case. However, before the actual runs were carried out an additional benchmarking study of the solver has been carried out, aiming in a better turbulence model selection for the final runs. For that reason a number of runs with the same solver were performed using the Zierke and Deutsch cascade geometry, a case study widely documented and available in the open literature. In this study the blades were at a positive incidence of +5 degrees, but the big availability of the experimental data makes this study suitable for the validation of numerical solvers. A detailed description of this cascade testing is given in Deutsch et al. (1987) whereas all the results from this study are presented within Deutsch et al., (1988). Work is done by the same authors on negative incidence angles but not for extreme values as the current study is focusing on (Deutsch et al., 1990).

A parametrical analysis regarding the turbulence modelling has been carried out by comparing the numerical 
results against the experimental data at three different positions downstream of the blades: $105 \%, 110 \%$ and $153 \%$. The capability of the solver to predict the wake propagation is verified in this way. The results obtained with all the different turbulence models are presented in Fig. 9, 10 and 11.

From the above plots it can be noticed that the $k-\varepsilon$ model demonstrates poor performance both in the center of the wakes as well as on the peripheral regions. This behavior of the $k-\varepsilon$ model can be observed at all the three locations analyzed. The $k-\omega$ SST model and the SSG model have a similar behavior in the peripheral zones, even if they estimate a lower velocity in respect to the experiments. But these two models behave in two different ways when they have to predict the central region of the wake. The Shear Stress Model is the one that predicts better the low velocity region both at $105.4 \%$ chord and at $152.5 \%$ chord. The BSL model has the same performance of the SST model at $105.4 \%$ chord but then its predictability degrades further downstream. Therefore, the turbulence model that can predict the most accurate separation pattern seems to be the Shear Stress Transport model and that was the applied turbulence closure scheme selected for this numerical study.

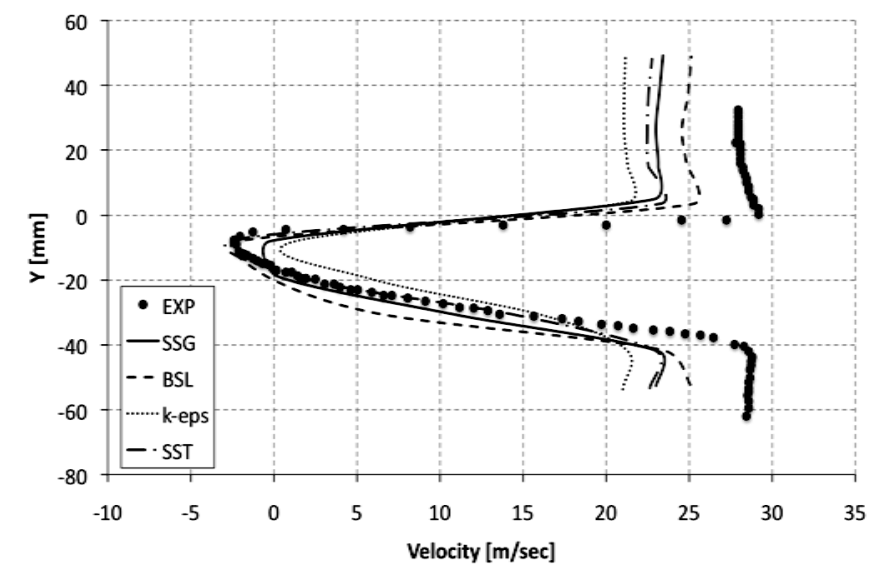

Figure 7 Wake profiles at $105.4 \%$ downstream.

Several computational grids, as summarised in Table 1 , have been created and tested in order the mesh sensitivity of the model to be assessed. Regarding the total number of elements of these three meshes, it is can be seen that a growth factor of about 1.5 between each other has been used. The most important regions in which the mesh has to be refined are: the region around the blade and the region just downstream of the blade. This is because the most relevant aspects of the phenomena are likely to happen almost exclusively in these regions. The results obtained with the three meshes have been compared in respect of total pressure loss coefficient and flow exit angle.

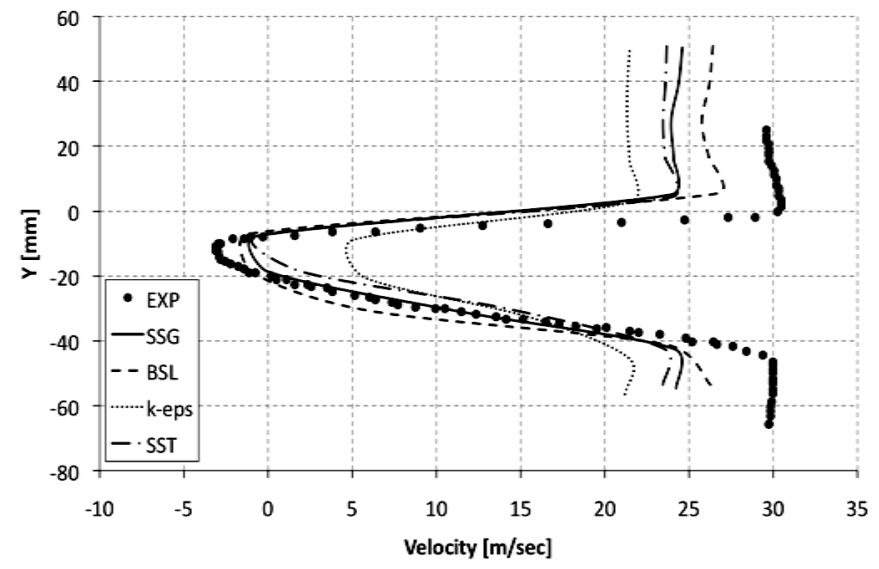

Figure 8 Wake profiles at $110 \%$ downstream.

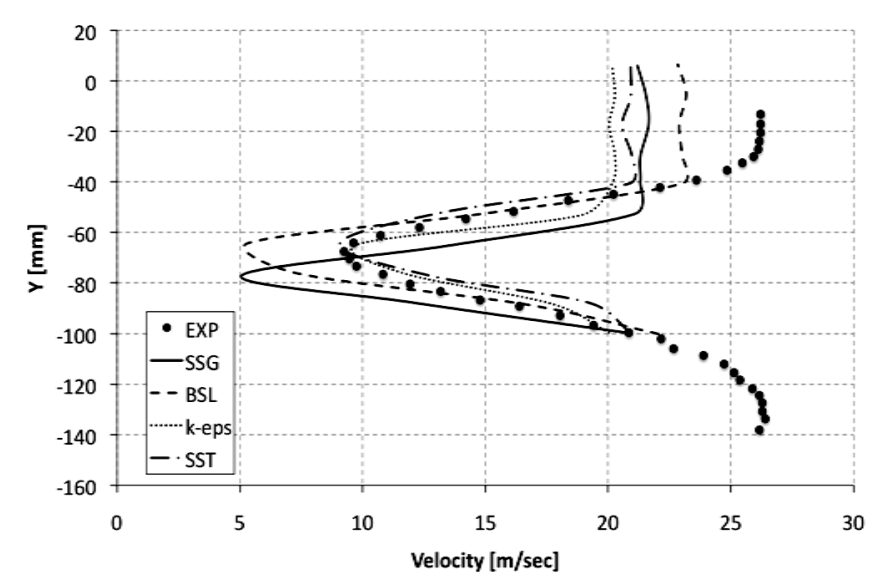

Figure 9 Wake profiles at $153 \%$ downstream.

The mesh sensitivity assessment showed that the three meshes produce results within a $0.5 \%$ range of difference, especially regarding the pressure loss. Relatively bigger deviation is observed at the hub region, with the medium and fine mesh to be closer to the experimental data. Therefore the medium mesh size has been selected as the most suitable one for this study and as a compromise between computational time and accuracy of the results.

Table 1 Mesh sizes used for the grid sensitivity assessment.

\begin{tabular}{ccccc}
\hline Grid & $\begin{array}{c}\text { Inlet } \\
\text { block }\end{array}$ & $\begin{array}{c}\text { Blade } \\
\text { block }\end{array}$ & $\begin{array}{c}\text { Plenum } \\
\text { block }\end{array}$ & $\begin{array}{c}\text { Total no. of } \\
\text { nodes }\end{array}$ \\
\hline 1 & 402,343 & $3,911,520$ & 956,087 & $5,269,950$ \\
2 & 511,576 & $6,984,456$ & $1,336,018$ & $8,832,050$ \\
3 & 645,719 & $9,577,920$ & $1,715,949$ & $11,939,588$ \\
\hline
\end{tabular}

Due to the extremely negative flow incidence it is highly possible unsteady effects to dominate the flowfield downstream of the blades. Therefore a number of unsteady numerical simulations have been carried out in order to evaluate whether their periodicity could be captured in 
average with a significantly faster steady state approach, since analysing the time dependency of those phenomena is not the major objective of that study.

Within this context, the time averaged unsteady results have been compared with those of the steady simulations in the span- wise as well as in the pitchwise direction as illustrated in Fig. 12 and 13.

As seen by the results, there is no difference between these two types of simulations for the points compared. The steady state results at various pitch- and spanwise positions are very close to the time averaged unsteady results, probably due to the act that the unsteadiness is highly periodic. Consequently, a very similar flow solution as far as the pressure loss through the cascade is concerned can be obtained equally well by the steady state numerical approach.

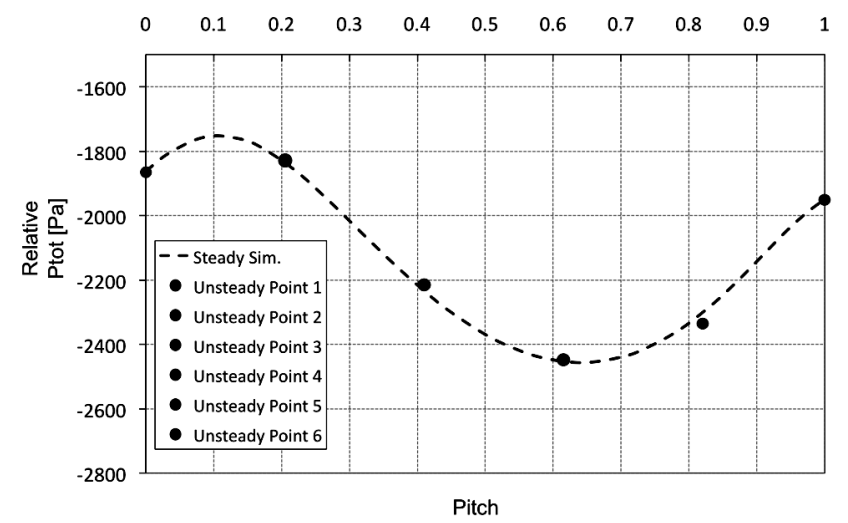

Figure 10 Comparison between steady and unsteady results in the pitchwise direction.

As the analysis of the unsteady effects is not the primary objective of this study, no time dependent numerical techniques have been used reducing also significantly the computational time with a minimum penalty on the quality of the results.

\section{RESULTS AND DISCUSSION}

The results from the numerical calculations are presented for the three main inlet Mach numbers, compared against experimental data for validation reasons. For comparing the results on the same basis, the built-in post processor of the numerical solver has not been employed. The flow data is directly exported from the simulation files and is further processed by a separate mass averaging script developed for this reason as described in Greitzer at al. (2004).

As expected, the flowfield is dominated by highly separated flows, as shown in Fig. 17, with a non-reattaching separation region propagating downstream until mixed with similar wakes from the channels nearby.
Comparative plots of the mass averaged total pressure loss (as defined in [20], eq.2), and flow outlet angle in the spanwise direction are presented in Figs. 14, 15 and 16 for three different inlet Mach numbers. As seen, all the pressure loss profiles are in good agreement with the experimental data with the highest deviation at the highest inlet Mach number simulation.

Regions of increased pressure losses can be observed at the hub and the tip, while the presence of the tip leakage vortices looks to affect a higher percentage of the blade span. A uniform pressure loss distribution between $10-70 \%$ of the blade span can be also highlighted in all cases indicating a less intense interaction of those flow layers with the hub and tip regions of high entropy generation rate.

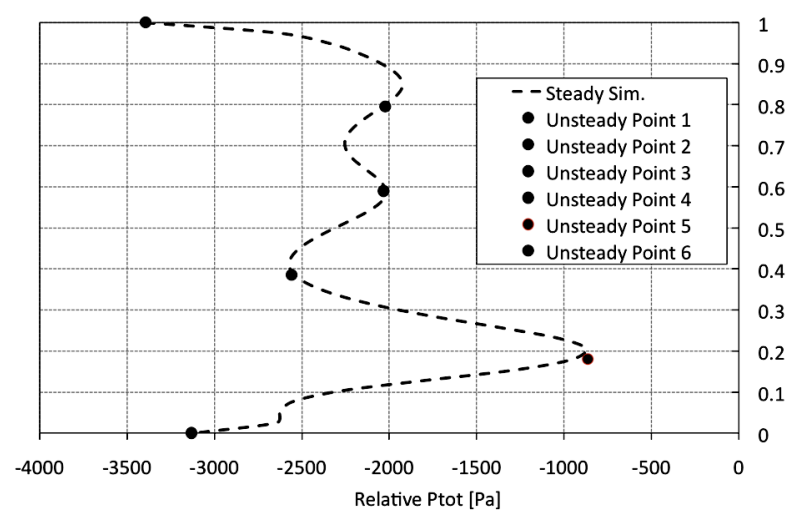

Figure 11 Comparison between steady and unsteady results in the spanwise direction.

As far as the comparison against the experimental data is concerned, the biggest deviation seems to exist at the hub region where the boundary layer thickness seems not well predicted by the solver. This happens probably due to the coarse grid definition at the hub region between the blades where most of the secondary flows start from. On the other hand, the tip losses are in good agreement, especially against the 5-hole probe which looks quite capable to capture the 3-dimensional flow phenomena at that region.

Regarding the flow exit angle, the numerical prediction is also in good qualitative agreement with the experimental data following the same trend in all cases with less than $8 \%$ deviation from the latter.

The difference in deflection angle can be partially explained using the difference also observed in the outlet velocity. For a defined control volume of one blade passage the mass conservation equation must be valid, therefore bigger deflection angles imply lower velocity. Obviously, the channel mass flow is slightly different between the numerical and experimental approach, however, the total pressure loss remains unaffected, since the results are expressed in a mass averaged way. 
As expected, the deflection angle's independency upon the inlet conditions can be also predicted by the solver as illustrated in Fig. 18 where the deflection angle distributions are presented for the entire range of inlet conditions tested.

Regarding the overall cascade performance prediction, the total pressure loss coefficient (defined as the massaveraged total pressure loss through the blades upon the inlet dynamic head) is presented in Fig. 18 in comparison to the experimental data. As seen from the plot, the weak dependency of the total pressure loss coefficient upon the inlet conditions can be also observed by the numerical results implying the not so strong independency of the flow from Reynolds number.

In addition, the loss coefficient is slightly under estimated by the numerical tool which may be due to the less losses predicted as shown at the bottom part of Figs. 14 to 16. As already mentioned, the experimental results have been obtained using a 3 - and a 5-hole pneumatic probe. However, regardless of their magnitude, 3-dimensional flow effects exist in any position due to the highly 3-dimensional blade geometry. Thus, due to the limited capability of the instrumentation to capture such phenomena, velocity components not in the yaw or pitch plane cannot be captured by the probes. Therefore, less total pressure downstream of the blades is recorded and consequently an increase in the total pressure loss is observed and this is more obvious regarding the 3-hole probe measurements.
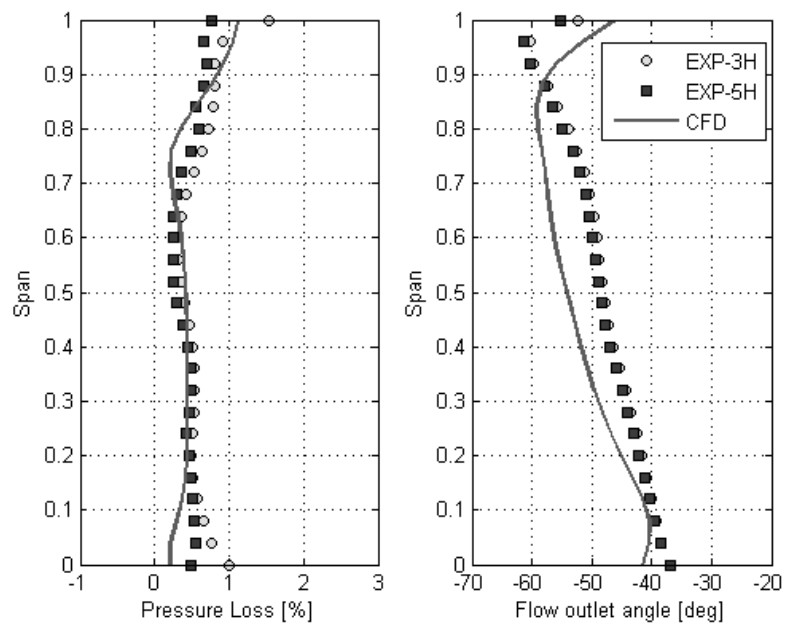

Figure 12 Spanwise loss and outlet angle for $\mathrm{M}=0.07$.
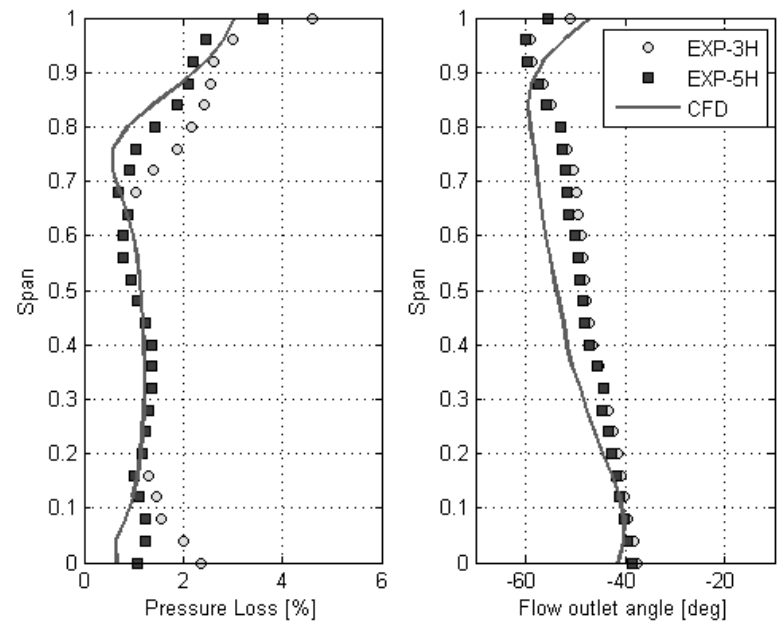

Figure 13 Spanwise loss and outlet angle for $\mathrm{M}=0.1$.
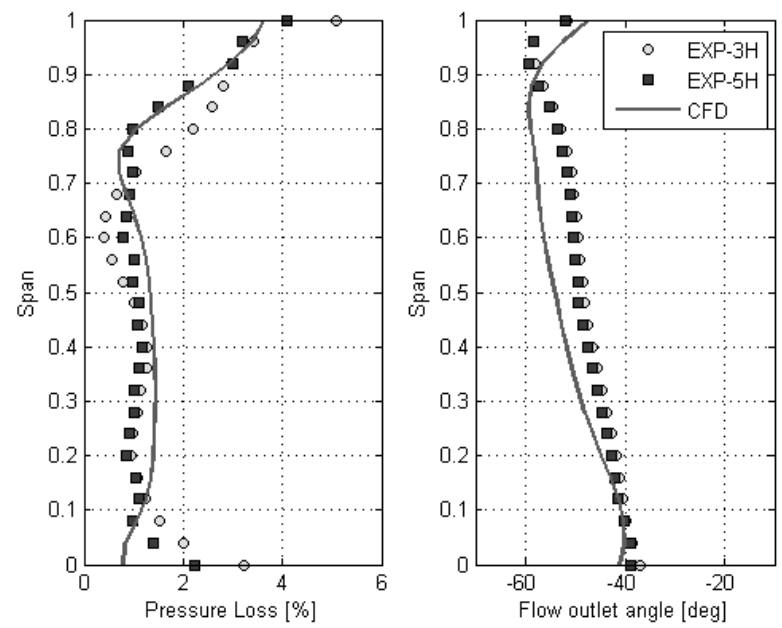

Figure 14 Spanwise loss and outlet angle for $\mathrm{M}=0.12$.

On the other hand, as can be also seen by the spanwise distributions of the flow properties, the numerical solver under estimates effects near the bottom wall. Moreover, a poor definition of the computational grid in the hub and top endwalls in the section between the blades results in an additional factor which limits the overall pressure loss in low levels, since the 3-dimensional viscous effects in that area are not appropriately predicted.

Consequently, the deviation between the measured and predicted total pressure loss for the current cascade is justified. Since it reasonable the solver to under-estimate the pressure loss while the testing provides wit over-estimated loss data, it can be securely claimed that the most realistic value of the overall energy loss is included within the abovedefined range of values as defined by the simulation and the experimental results that is between 1.4 and app. 1.45 in overall. 


\section{CONCLUSIONS}

In the current work, the flow through a compressor cascade at highly negative incidence angle has been experimentally and numerically investigated. Design details of the test facility, as well as blade geometrical features have been also presented. The test program followed before and during the flow measurements is described. The flow conditions are explained whereas results regarding the total pressure loss through the cascade are presented as a function of the span of the blade for each inlet Mach number tested. The overall cascade properties (total pressure loss coefficient and flow exit angle) are summarized as a function of the inlet flow conditions. A CFD model for predicting the flowfield through the blades has been developed and validated against the experimental data acquired. Details about the meshing strategy, the boundary conditions and the turbulence closure used have been also presented. An assessment of the unsteadiness of the flow has been carried out, which showed that the phenomena are highly periodical. Thus, an unsteady simulation approach is not required if the model is to be validated against overall performance properties such as pressure loss. The spanwise predicted distributions of pressure loss and deflection angle are compared against the experimental results. In addition, the overall cascade performance at high incidence (total pressure loss and total pressure loss coefficient) is presented as a function of the inlet conditions, validated against the experimental values as well.

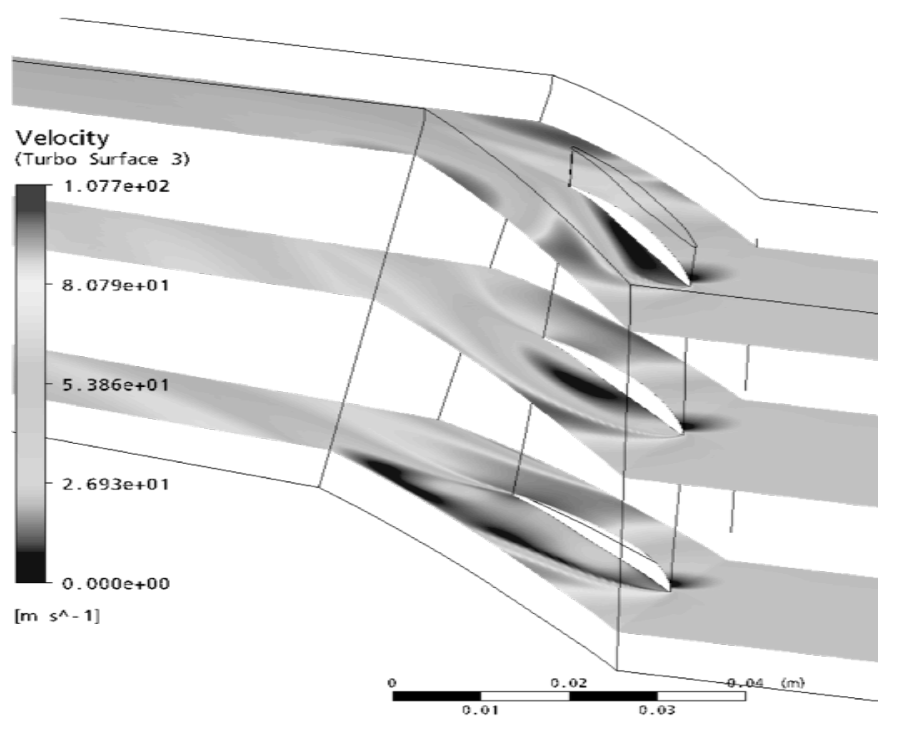

Figure 15 Flow separation structures at different positions along the span.
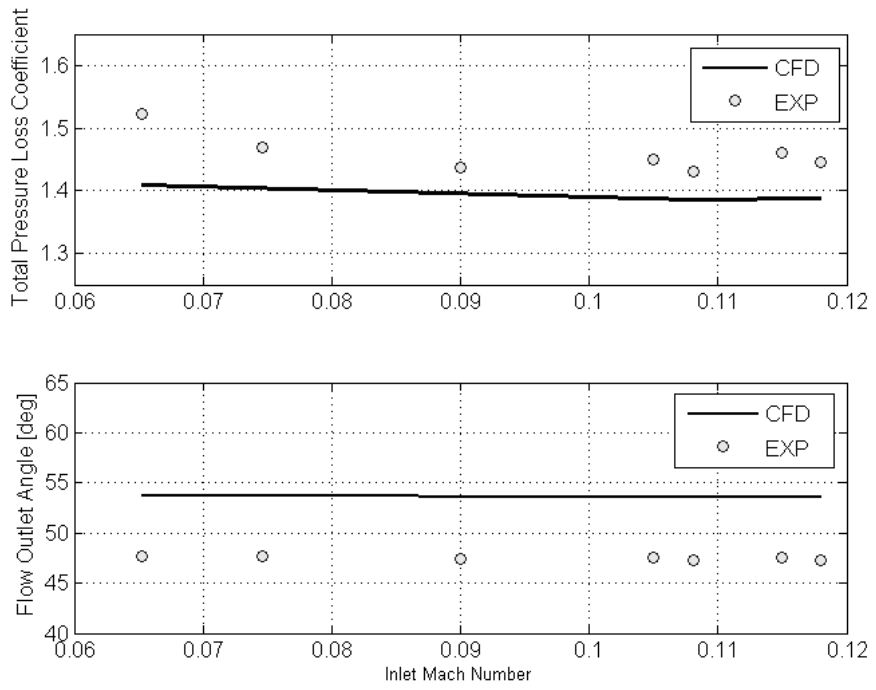

Figure 16 Overall cascade performance as a function of the inlet conditions.

The main conclusions from this study can be summarized as follows:

- The compressor cascade at -58 degrees mid-span incidence angle experiences a total pressure loss coefficient of 1.4- 1.5 weakly dependent upon the inlet flow Mach number.

- The spanwise distributions of the total pressure loss indicate higher losses at the tip of the blade than at the hub region caused by the tip leakage effects.

- The CFD model looks capable enough to capture the flow phenomena struggling only at the hub region. A more complex hub endwall mesh definition may improve the quality of the results in this direction.

- The validation against the experimental data has shown that the pressure loss and deflection angle distributions in the spanwise direction are predicted with less than $8 \%$ error. However, considering that this is the first attempt to model so extreme off-design blade operation, the $8 \%$ deviation provides confidence for higher accuracy in future similar studies while indicates the challenges for a future numerical prediction of even higher fidelity.

- In overall, the confidence on the highly separated flows prediction is enhanced by the validation against the experimental data, thus numerical models of similar structure can be utilised for further research. 


\section{REFERENCES}

1. Abernethy, R., B., Benedict, R., P., Dowdell, R., B. (1985). ASME measeurement uncertainty. Journal of Fluids Engineering, 107, 161-164.

2. Alexiou, A., Baaldbergen, E., Mathioudakis, K., Kogenhop, O., Arendsen, P. (2007). Advanced capabilities for gas turbine engine performance simulations. ASME Turbo Expo, Montreal, Canada.

3. Carter, A. (1950). Low-speed performance of related aerofoils in cascade. $A R C, 29$.

4. Chaluvadi, V., Kalfas, A., Hodson, H., Ohyama, H., Watanabe, E. (2003). Blade row interaction in a high pressure steam turbine. ASME Journal of Turbomachinery, 125, 014-024.

5. Deutsch, S., Zierke, W. (1986). The measurement of boundary layers on a compressor blade in cascade at high positive incidence angle. 1-Experimental techniques and results. NASA-CR-179491.

6. Deutsch, S., Zierke, W. (1987). The measurement of boundary layers on a compressor blade in cascade. Part 1- A unique experimental facility. ASME Journal of Turbomachinery, 109, 520-526.

7. Deutsch, S., Zierke, W. (1988). The measurement of boundary layers on a compressor blade in cascade. Part 2- Suction surface boundary layers. ASME Journal of Turbomachinery, 110, 138-145.

8. Deutsch, S., Zierke, W. (1988). The measurement of boundary layers on a compressor blade in cascade. Part 3- Pressure surface boundary layers and the near wake. ASME Journal of Turbomachinery, 110, 146-152.

9. Deutsch, S., Zierke, W. (1990). The measurement of boundary layers on a compressor blade in cascade. Part 4- Flowfields for incidence angles of -1.5 and -8.5 degrees. ASME Journal of Turbomachinery , 112, 241255.

10. Dixon, S. (1998). Fluid mechanics and thermodynamics of turbomachinery.

11. Ekaterinaris, J., Menter, F. (1994). Computation of Separated and Unsteady Flows with one and twoequation turbulence models. AIAA 32nd Aerospace Sciences Meeting and Exhibit, Reno, Nevada .

12. Förster W., Karpinski G., Krain H., Röhle I., Schodl R. (2000). 3- Component-Doppler-Laser-Two-Focus Velocimetry Applied to a Transonic Centrifugal Compressor. 10th International Symposium on "Application of Laser Techniques to Fluid Mechanics“, Lisbon.

13. Gallimore, S. (1998). Axial flow compressor design. Proceedings of I.Mech.E. , 213.

14. Gallimore, S. (1998). Viscous throughflow modelling of axial compressor blade rows using a tangential blade force hypothesis. Journal of Turbomachinery , 120, 662670.

15. Gostelow, J. (1984). Cascade aerodynamics. Pergamon Press.
16. Greitzer, E., Tan, C., Graf, M. (2004). Internal flow concepts and applications.

17. Horlock, J. (1973). Axial flow compressors. Fluid mechanics and thermodynamics.

18. Howell, A. (1945). Design of axial compressors. Proceedings of IMechE , 153.

19. Howell, A. (1942). The present basis of axial flow compressor design: Part I, Cascade theory and performance. ARC R and M , 2095.

20. Johansen, E., S., Allen, R., D., Rediniotis, O., K., Zeiger, M., D. (2003). Embedded sensor, fast response, multi-hole probes. $41^{\text {st }}$ Aerospace Sciences Meeting and Exhibit, Reno, Nevada.

21. Korakianitis, T. (1993). On the propagation of viscous wakes and potential flow in axial-turbine cascades. Journal of Turbomachinery, 115, 118-127.

22. Kurzke, J. (1996). How to get component maps for aircraft gs turbine performance simulations. International Gas Turbine and Aeroengine Congress and Exhibition, Birmingham, UK.

23. Lieblein, S. (1965). Experimental flow in twodimensional cascades. NASA SP-36, Chapter VI .

24. Lieblein, S. (1959). Loss and stall analysis of compressor cascades. Transactions of ASME, Series $D, 81$.

25. Lieblein, S., Roudebush, W. (1956). Theoretical loss relation for low-speed 2D cascade flow. NACA T.N. 3662 .

26. Menter, F. (1994). Two-equation eddy-viscosity turbulence models for engineering applications. AIAA Journal , 32.

27. Menter, F. (1993). Zonal two equation k- $\omega$ Turbulence Models for Aerodynamic Flows. AIAA 24th Fluid Dynamics Conference, Orlando, Florida .

28. Novak, R. A. (1967). Streamline curvature procedures for fluid-flow problems. 89, 478-490.

29. Pachidis, V., Pilidis, P., Talhouarn, F., Kalfas, A., Templalexis, I. (2006). A fully integrated approach to component zooming using computational fluid dynamics. ASME Journal of Engineering for Gas Turbines and Power, 128.

30. Pollard, D., Gostelow, J. (1967). Some experiments at low speed on compressor cascades. Journal of Engineering for Power , 89 (3), 427-436.

31. Ramakrishna, P., V., Govardhan, M. (2009). Aerodynamic performance of low speed axial flow compressor rotors with sweep and tip clearance. Engineering Applications of Computational Fluid Mechanics 3 (2), 195-206.

32. Riegler, C., Bauer, M., Kurzke, J. (2000). Some aspects of modeling compressor behavior in gas turbine performance calculations. ASME Turbo Expo, Munich, Germany.

33. Schodl R., A (1975) Dual focus velocimeterv for turbomachine applications. Von Karman Institute, Lecture Series 78, p. 39. 
34. Sieverding, C. (1985). Aerodynamic development of axial turbomachinery blading. Thermodynamics and fluid mechanics of turbomachinery, 1, 513-565.

35. Todd, K. (1947). Practical aspects of cascade windtunnel research. Proceedings of IMechE , 157.

36. Ummiti, M., B., Sitaram, N., Prasad, B., V., S., S., S. (2009). Computational investigation of effect of axial spacing on blade row interaction in a $1^{1 / 2}$ stage axial flow turbine. Engineering Applications of Computational Fluid Mechanics, 3 (1), 56-70.

37. Wernet M., P., Bright M., M., Skoch G., J. (2001). An Investigation of Surge in a High Speed Centrifugal Compressor Using Digital PIV. Trans. of ASME, Journal of Turbomachinery, 123, 418-428.
38. Wilcox, D. (1988). Reassessment of the scaledetermining equation for advance turbulence models. AIAA Journal , 26.

39. Zachos, P., Pachidis, V., Charnley, B., Pilidis, P. (2009). Flowfield investigation of a compressor cascade at high incidence-Part I: Pneumatic probe measurements. ASME Turbo Expo, Orlando, Florida.

40. Zachos, P., Pengue, F., Pachidis, V., Pilidis, P. (2009). Flowfield investigation of a compressor cascade at high incidence-Part II: Numerical analysis. ASME Turbo Expo, Orlando, Florida. 